\title{
I connettivi perché e weil come introduttori di enunciati autonomi nei giornali di oggi. Un'analisi corpus-based
}

\author{
Benedetta Rosi \\ Universität Basel
}

\begin{abstract}
The aim of this paper is, first of all, to outline the contemporary uses of causal structures in Italian writing, focusing on some less canonical syntactic and textual manifestations. These non-prototypical constructs will be, then, compared with the corresponding ones in German. The analysis that will be presented are based on qualitative and quantitative data derived from corpora of Italian and German newspaper writing. As far as the Italian language, the paper will focus on the causal connective perché: after presenting its frequency of use compared to the other causal connectives, we will discuss some of its most common syntactic and informative-textual configurations, with particular reference to the case in which perché introduces an independent utterance, preceded by a strong punctuation mark. Right this last use will be observed also in German newspapers, thanks to a preliminary survey that will focus on the causal connective weil. For both languages we will consider the frequency of this peculiar use, as well as its possible forms and textual functions, in order to highlight analogies and differences between German and Italian.
\end{abstract}

Keywords: connectives, punctuation, contrastive analysis (Italian-German), newspaper writing

\section{Introduzione}

Col presente contributo si intende ritornare su alcune manifestazioni dei costrutti causali nell' italiano contemporaneo (cfr. anche Rosi 2018 e Rosi \& Ferrari 2019), aprendo anche a riflessioni in ottica contrastiva con il tedesco. Il terreno d'indagine di tale confronto interlinguistico sarà costituito dalla scrittura di tipo giornalistico, definita a più riprese varietà in cui si manifesta in modo più evidente la lingua media scritta di oggi (cfr. Bonomi 1993; Antonelli 2011) o, in altri termini, 
varietà che «unmittelbarer den Sprachzustand ihrer Zeit [spiegelt], als es jedes andere gedruckte Medium vermag» ${ }^{1}$ (Lüger 1983: 23).

Più in particolare, all'italiano sarà dedicata la prima parte del lavoro. Ripercorrendo i risultati ricavati da una ricerca corpus-based incentrata sulle strutture causali nell'italiano scritto contemporaneo (Rosi 2019), si mostrerà come per alcuni fenomeni l'uso delle causali nei testi funzionali indagati presenti meno divergenze di quanto si sarebbe potuto attendere rispetto a quello degli stessi costrutti nel parlato. L'attenzione sarà rivolta ai nessi introduttori, e soprattutto a perché (\$2.1), alle configurazioni sintattiche e informativo-testuali a esso associate ( $\$ 2.2$ ), con particolare riferimento al caso meno prototipico in cui il connettivo apre un enunciato autonomo, essendo preceduto da un segno di punteggiatura forte $(\S 2.3)$.

Proprio quest'ultima manifestazione della causale sintatticamente e testualmente autonoma sarà al centro del confronto con il tedesco, a cui sarà dedicata la seconda parte del contributo ( $\$ 3)$. Caso paradigmatico saranno le frasi aperte dal connettivo weil, equivalente funzionale dell'italiano perché. Obiettivo di questo sondaggio contrastivo sarà verificare se marcature interpuntive più estreme e riconducibili a ragioni di essere puramente comunicative siano presenti anche nei giornali di una lingua in cui, sulla base di quanto stabilito dalla norma (cfr. ad esempio Duden 2018), l'interpungere tende a seguire in generale un più rigido fondamento morfosintattico.

\section{I costrutti causali nell'italiano di oggi. Un'analisi nei giornali}

Gli studi sulla sintassi della frase complessa nel parlato spontaneo hanno messo in evidenza a più riprese come, per via delle proprietà intrinseche di questa varietà diamesica, la subordinazione avverbiale si caratterizzi in generale per una "scarsa dispersione" di forme (Berretta 1994: 251). Nell' ambito della causalità, ciò si traduce in una preferenza quasi esclusiva per il connettivo perché nella sua tipica distribuzione post-reggente, a scapito delle svariate alternative esistenti a livello di sistema ${ }^{2}$. Dunque, il parlato anziché affidarsi alla variazione lessicale, che implica tipicamente anche variazione semantica, sceglie di sfruttare un unico mezzo, caratterizzato però da un'ampia polifunzionalità (Voghera 2017: 114). A livello

\footnotetext{
1 Trad.: 'rispecchia in modo più diretto di qualsiasi altro medium la situazione linguistica coeva'.

${ }^{2}$ La preferenza netta di perché in questa distribuzione e la quasi assenza delle sue alternative è quantificata dai rilevamenti corpus-based presentati in Acciardi (2010) e in Rosi (2019): nel parlato spontaneo informale (corpus DB-IPIC: 125.000 parole; online: http://lablita.it/app/dbipic/index.php?corpus=; cfr. Panunzi \& Gregori 2012) il dato si aggira intorno al $90 \%$.
} 
funzionale, infatti, perché introduce non soltanto "vere" relazioni sintattico-semantiche di subordinazione, ma piuttosto pseudo-subordinazioni: il contenuto introdotto da perché si presenta come isolato in un enunciato - o in un turno - indipendente rispetto a quello in cui si colloca la reggente, da cui è separato da confini intonativi terminali (cfr. anche Cresti 2012). Non sono rari inoltre esiti ancora più estremi in cui il connettivo si comporta come un semplice segnale discorsivo, con il valore di marcare la conquista del banco conversazionale da parte del locutore o l'inizio del turno (Moeschler 1987).

Mantenendo le tendenze delineate brevemente per l'italiano parlato come termine di paragone, si passi ora al polo opposto dell'asse diamesico: alla scrittura funzionale controllata, con particolare riferimento alla lingua dei giornali. Si tratta di una varietà che dovrebbe essere frutto di un'opera di pianificazione più attenta, da cui ci si potrebbe aspettare un grado di elaborazione morfosintattica e lessicale più alto, e dunque un maggiore sfruttamento della varietà di forme che l'italiano ha a disposizione a livello di sistema. In realtà, una ricerca corpus-based (Rosi 2019) incentrata sulle strutture causali proprio nella stampa contemporanea ha consentito di osservare che in questi testi si riscontrano per certi aspetti differenze meno nette e significative rispetto al parlato di quanto si sarebbe potuto attendere: in particolare per quanto riguarda la varietà di introduttori subordinanti, la distribuzione sintattica di reggente e causale e alcuni peculiari sfruttamenti testuali di quest'ultima; fenomeni su cui si soffermeranno i paragrafi seguenti.

Più precisamente, $\mathrm{i}$ dati a cui si fa riferimento sono stati ricavati dalla sezione di prosa giornalistica di un corpus già esistente di italiano scritto funzionale di registro medio-alto indicato con la sigla PUNT-IT. Il sotto-corpus qui considerato (320.000 parole) include articoli (cronaca, politica, rubriche di sport o spettacoli, editoriali) tratti da quotidiani generalisti (La Repubblica, Corriere della Sera, La Stampa; 220.000 parole), locali (Messaggero Veneto, Il Tirreno, La Nuova Sardegna; 40.000 parole), free-press (Leggo; 10.000 parole) e periodici (Espresso; 50.000 parole), e a livello diacronico rappresenta 1'italiano giornalistico degli ultimi venti-trent' anni circa ${ }^{3}$.

\footnotetext{
${ }^{3}$ Più in particolare il corpus PUNT-IT è stato compilato nel 2015 all'Università di Basilea nell'ambito del progetto di ricerca FNS Le funzioni informativo-testuali della punteggiatura italiana contemporanea, tra sintassi e prosodia, i cui risultati sono ora pubblicati in Ferrari et al. (2018). PUNT-IT comprende complessivamente 520.000 parole, e oltre alla sezione di testi giornalistici, sopra descritta e scelta per questa indagine, include testi saggistici di tipo accademico (150.000 parole) e testi giuridico-amministrativi (50.000 parole). L'arco di tempo coperto complessivamente è di circa trent'anni (i testi sono infatti stati pubblicati tra il 1985 al 2015) e intende essere rappresentativo della scrittura italiana contemporanea.
} 


\subsection{Perché tra gli altri introduttori causali}

Considerando le sole strutture causali esplicite - nettamente più rappresentative nei giornali indagati di quelle implicite (circa $90 \%$ vs $10 \%$ dei casi) - un risultato meno atteso riguarda senz'altro la gamma di introduttori individuati. Nell'ambito della frase complessa, a livello di sistema-langue l'italiano dispone di una ricchissima serie di connettivi associati alla semantica causale: perché, poiché, visto che, siccome, in quanto, dal momento che, dato che, atteso che, posto che, considerato che, essendo che, giacché, per il fatto che, tanto più che. Ciononostante, a livello di uso dall'analisi corpus-based è emersa una diversificazione decisamente bassa: addirittura nell' $80 \%$ delle occorrenze si opta per perché, dato che equivale a circa 430 unità in termini assoluti. Gli altri introduttori si spartiscono il restante $20 \%$ dei casi, con le preferenze messe in evidenza dal seguente grafico:

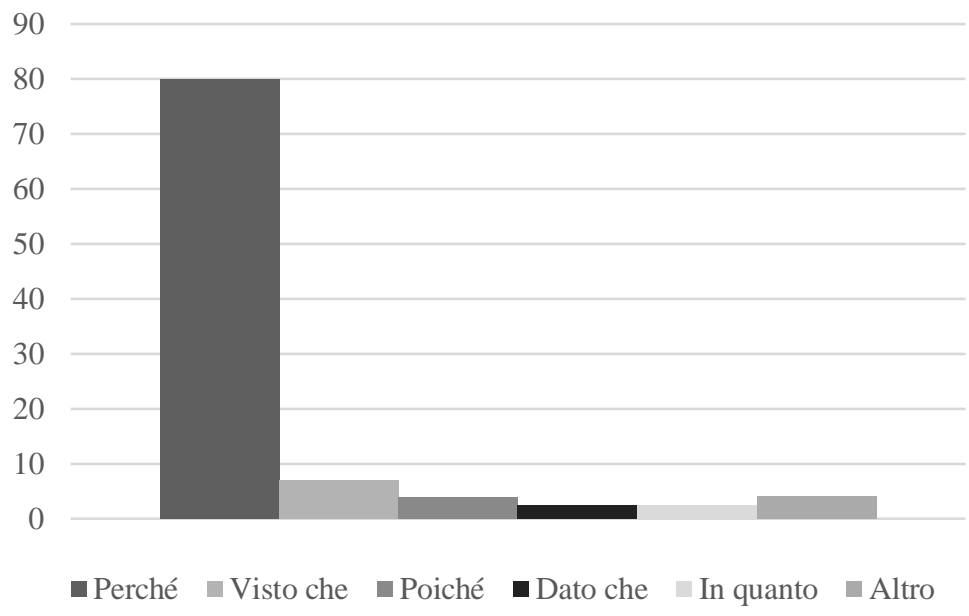

Figura 1. Tipi di introduttori causali

Dunque, tra le alternative a perché figurano, nell'ordine, visto che (7\%), poiché (4\%), dato che e in quanto (tra il 2 e il 3\%); altri connettivi causali (dal momento che, siccome, tanto più che, giacché, posto che: complessivamente il $4 \%$ dei casi rimanenti) compaiono in modo persino più sporadico; altri ancora (come atteso che, considerato che, per il fatto che) sono del tutto assenti. Come si nota, lo scarto tra i dati relativi ai due tipi di causali più frequenti - quella con perché e quella con visto che - è altissimo, corrispondendo a più del $70 \%$. 
Si passi a commentare i dati ricavati. Non è il fatto che perché sia il connettivo più usato a destare stupore, bensì la sua frequenza d'uso: il quadro che si ricava infatti appare addirittura molto vicino a quello tratteggiato per il parlato spontaneo ${ }^{4}$. Se però in quest'ultimo caso percentuali così nette sono attese per via del minimalismo di forme tipico di questa varietà, esse risultano invece meno prevedibili in una scrittura pianificata e di registro medio-alto come quella indagata. Pur considerando la tendenza alla semplificazione sintattica e lessicale che investe ormai da decenni la lingua dei giornali (cfr. Bonomi 2002), ci si sarebbe aspettati una maggiore variazione lessicale, soprattutto alla luce della ricchezza di mezzi a disposizione dell'italiano per veicolare il significato causale tramite la subordinazione, a cui viene dedicato ampio spazio nella trattazione delle grammatiche tradizionali. In particolare, a colpire non è l'assenza o la scarsa presenza di introduttori come giacché, atteso che o posto che, i quali sono riconducibili a registri più formali o a varietà di scrittura di tipo diverso (nel caso degli ultimi due, ad esempio, a quella regolativo-amministrativa), ma quella di connettivi come siccome, dal momento che, dato che, che non sono marcati diafasicamente rispetto a perché e sono definiti spesso come di largo uso anche o proprio nei giornali (cfr. ad esempio Serianni 1989: 576-577).

Le ragioni della diffusione di perché a scapito di ogni altra opzione sono da ricondurre alla sua versatilità a livello informativo-testuale (cfr. infra) e semantico-concettuale. Per quanto riguarda quest'ultimo aspetto, diversamente da quanto accade con altri connettivi causali, perché si presta grazie alla sua semantica lessicale a veicolare diversi tipi di relazione causale. Con lo stesso mezzo linguistico si possono esprimere a seconda del contesto due tipi di legami causali. i) Si hanno relazioni causali in senso stretto, instaurate cioè tra gli eventi evocati nel testo: si possono distinguere cause, se l'evento non prevede il controllo da parte di un agente (La strada è allagata perché ha piovuto), o motivi, se lo prevede (Ha commesso il reato perché era disperato) (cfr. Previtera 1996). ii) Oppure legami di motivazione esplicativa/argomentativa, ovvero relazioni di composizione testuale attraverso cui lo scrivente organizza il proprio pensiero all'interno del testo (Non è ancora a casa. La macchina non è nel vialetto; Piove? Perché vorrei uscire). In linea con Sweetser (1990) e Ferrari (1995), per i legami del primo tipo parliamo di relazioni che si agganciano al contenuto proposizionale della reggente, mentre per quelli del secondo tipo di relazioni che si agganciano all'atteggiamento epistemico o illocutivo.

A livello sintattico, nel caso i) perché introduce legami di vera subordinazione: la causale risulta integrata nella reggente; mentre nel caso ii) siamo di

\footnotetext{
${ }^{4}$ Cfr. anche la nota 2.
} 
fronte a pseudo-subordinazioni o subordinazioni testuali (Berretta 1994: 252; Ferrari et al. 2008): il connettivo assume un valore pragmatico, introducendo un'unità indipendente, non integrata alla reggente ma giustapposta.

Nel corpus il connettivo è effettivamente usato con entrambi i valori, preferendo l'uno o l'altro anche a seconda del tipo di testo: sia cioè in articoli di tipo informativo, narrativo o descrittivo, in cui si vuole indicare la causa di un evento o il motivo di un' azione compiuta intenzionalmente, sia in editoriali o altri articoli di carattere argomentativo, per introdurre ragionamenti o spiegazioni.

\subsection{Le manifestazioni informativo-testuali delle frasi con perché}

A livello sintattico-distributivo, nell'enunciato che accoglie il costrutto la causale ${ }^{5}$ risulta più frequentemente posposta rispetto alla reggente ( $v s$ anteposta o inserita al suo interno). A influire su questa tendenza complessiva è il dato relativo a quella introdotta da perché, che segue la reggente addirittura nel $98 \%$ dei casi, in linea con quanto accade per il parlato.

In questa posizione sintattica il connettivo perché nei giornali si inserisce in un paradigma di configurazioni informativo-testuali molto variato, con alcune preferenze. L'analisi ha mostrato che, contraddicendo il principio di isomorfismo tra gerarchia sintattica e gerarchia informativa (cfr. Ferrari 2016), la frase con perché costituisce molto spesso un' informazione comunicativamente rilevante, in grado di far progredire il testo a livello tematico o logico. Tale rilievo risulta evidente in casi come (1), in cui è soltanto la causale a portare avanti l'architettura semantico-pragmatica del testo, visto che la reggente è testualmente data:

(1) Soprattutto, Putin è figlio del decennio eltsiniano.

[...] Putin è figlio di Eltsin perché è la sua Russia che il candidato Presidente ora riceve in eredità.

(PUNT-IT_254_Corriere_della_Sera_26.03.2000_Editoriali)

La reggente svolge principalmente la funzione di aggancio tematico nei confronti del cotesto sinistro, limitandosi a ripetere quasi letteralmente quanto appena espresso; è la causale a contenere l'informazione nuova e più importante. A livello sintattico, siamo qui sempre di fronte a subordinazioni in senso stretto.

\footnotetext{
${ }^{5} \mathrm{Si}$ sceglie di continuare a utilizzare il termine "(subordinata) causale" (e parallelamente quello di "reggente") in senso ampio, per indicare sia la manifestazione del costrutto che realizza una vera subordinazione e introduce un legame causale in senso stretto sia quella che costituisce una pseudo-subordinazione e introduce un legame argomentativo-esplicativo.
} 
Il rilievo comunicativo si manifesta inoltre in esempi come (2), in cui reggente e causale veicolano due contenuti informativi distinti ma entrambi focalizzati, risultando così paritarie in termini di importanza comunicativa:

(2) Vite Nuove di Ingo Schulze è un monumentale romanzo sulla riunificazione della Germania. Anzi è "il" monumentale romanzo, perché nessun altro scrittore tedesco si è cimentato come lui a raccontare che cosa pensavano, che cosa sentivano i tedeschi dell'Est; come si viveva con i sentimenti giusti nello Stato sbagliato; e che cosa attraversò le loro menti e i loro cuori in quei mesi subito prima e subito dopo la caduta del Muro. Quei mesi che erano cominciati con le marce di protesta, ogni lunedì sera, a Lipsia, quando la gente, sconfiggendo la paura della Stasi [...].

(PUNT-IT_312_Repubblica_18.08.2007_CultSpett)

In particolare, la reggente compie un atto di rettifica autonomo nei confronti del cotesto precedente: il connettivo anzi esaurisce cioè il campo della sua azione semantica ai limiti della proposizione. La causale, dal canto suo, veicola un contenuto altrettanto nuovo e rilevante per l'organizzazione logico-tematica del resto del paragrafo, tanto più che viene argomentato ampiamente nel cotesto susseguente. A livello sintattico, si possono avere con questa configurazione informativa anche pseudo-subordinazioni.

L'interpretazione sintattico-informativa ha ricadute sull'uso interpuntivo: la virgola tra le due proposizioni tende a occorrere solo quando reggente e causale hanno funzioni informative distinte come in (2), fungendo da indizio di divergenza informativa (cfr. Ferrari \& Rosi in stampa per un approfondimento), e non in casi come (1), in cui il costrutto è più compatto informativamente presentando il focus soltanto nella subordinata. I livelli di analisi informativo e interpuntivo si intrecciano inevitabilmente anche con quello semantico, mostrando le seguenti tendenze: la relazione causale si aggancia al contenuto proposizionale quando il costrutto è più compatto informativamente $\mathrm{e}$ interpuntivamente (1); per agganciarsi all'atteggiamento epistemico o illocutivo è necessario invece che vi sia divergenza informativa (2).

\subsection{Perché come introduttore di enunciati autonomi}

Come mostrato nel paragrafo precedente, perché post-reggente si caratterizza spesso per introdurre contenuti informativamente importanti, in primo piano nell'enunciato o nel testo. Il rilievo comunicativo diviene massimo quando il connettivo apre un enunciato autonomo, essendo preceduto da un segno di punteggiatura forte (punto, due punti o punto e virgola), come nel seguente esempio: 
(3) Oltre 10 mila specie di vertebrati sono state messe sotto osservazione dal 1970 al 2010: in media, il calo è stato del 52 per cento. Un esempio su tutte, la tigre ridotta a 3 mila esemplari. [...] L'esempio della tigre non è fatto a caso. Perché a pagarla cara sono soprattutto i predatori. Nelle reti alimentari occupano la posizione apicale, hanno specifiche ed elevate esigenze metaboliche [...]. Va da sé che sono le specie che più rapidamente vanno in crisi.

(PUNT-IT_156_Corriere_della_Sera_01.10.2014_Cronaca; in Rosi 2018)

Che nella scrittura giornalistica si possano trovare simili emarginazioni frasali è già stato osservato in diversi studi su questa varietà. Si tratterebbe, in particolare, di una delle forme del cosiddetto «periodare monoproposizionale» (cfr. almeno Bonomi 2002: 243-248): accanto a enunciati saturati da frasi indipendenti semplici, complete sintatticamente ma molto brevi, si hanno enunciati esauriti da frasi coordinante (introdotte da $e, m a, o$ ) o subordinate - come nel nostro caso o persino da singoli sintagmi. La ricerca condotta ha consentito, grazie ai dati corpus-based, di quantificare la diffusione del fenomeno con le frasi introdotte da perché: in particolare, è emerso che quasi un quarto delle causali in posizione posposta aperte dal connettivo è preceduto da un segno interpuntivo forte. Si tratta di un dato significativo, il quale conferma che alla marcatezza sintattico-testuale del costrutto non corrisponde marcatezza in termini di frequenza; non si tratta cioè di un uso legato solo allo stile di pochi giornalisti.

Sebbene più raramente, esempi simili sono stati individuati anche con altri connettivi di ambito causale, come tanto più che o visto che:

(4) Comprensibile che Ferrandino sia irritato, visto che la stagione turistica è alle porte. Tanto più che le cose stanno già andando maluccio [...]. (PUNTIT_L'Espresso_28.04.2010) ${ }^{6}$

(5) Giornali e tele-giornali. Opposti fra loro. Visto che le informazioni in tivù, in molte reti, sono filtrate.

(PUNT-IT_100_Repubblica_28.09.2009_Editoriali)

In tutti i casi, tale strategia ha ragioni di essere puramente comunicativo-testuali: attribuire alla causale un rilievo comunicativo elevato, pari a quello della reggente

\footnotetext{
${ }^{6}$ Un'indagine a scopo di verifica condotta sulla locuzione causale tanto più che nella sezione Stampa del corpus CORIS (Università di Bologna) ha messo in evidenza come questo connettivo sia addirittura separato dalla reggente più facilmente per mezzo del punto (70\% dei casi) che non della virgola (30\%) (cfr. sempre Rosi 2019).
} 
(cfr. Ferrari 2016). Entrambe le frasi risultano infatti focalizzate e possono interagire in modo indipendente con il cotesto. Ciò è particolarmente utile soprattutto quando si vuole tematizzare ulteriormente l'informazione contenuta dalla causale negli enunciati successivi, dando vita ad argomentazioni ampie, che si estendono per più enunciati e acquistano così particolare importanza nell'architettura logico-referenziale del testo, come nel caso di (3), proposto in precedenza, o di (6):

(6) [...] è giusto stringersi attorno alla nazione americana, dopo le terribili esplosioni dell'11 settembre. Anche perché quei morti ci riguardano, ci toccano molto da vicino. Era gente come noi, con un mestiere qualunque, in una giornata qualunque. Non militari in trincea [...]. (PUNTIT_124_Stampa_15.09.2001_Editoriali)

Qui il tema introdotto da perché diviene centrale nel cotesto successivo, che lo motiva, lo argomenta ulteriormente.

Sempre a livello interpretativo, si riconoscono due possibilità (cfr. anche Ferrari 1995). Da un lato si hanno occorrenze in cui il segno forte collocato prima di perché si inserisce in un costrutto altrimenti potenzialmente coeso a livello sintattico e semantico-informativo, come in (7):

(7) Ammazzato domenica pomeriggio tra la gente a passeggio [...], con una coltellata sotto lo sterno. Soltanto perché era un homeless, un senzatetto. (PUNT-IT_133_Corriere_della_Sera_18.04.2000_Cronaca; in Rosi 2018)

In questo testo, se tra reggente e subordinata non fosse presente alcun segno interpuntivo, il costrutto potrebbe facilmente essere interpretato come compatto informativamente e integrato sintatticamente (è stato ammazzato soltanto perché era un homeless); e per quanto riguarda il tipo di relazione semantica, saremmo qui di fronte a legami causali in senso stretto (cause o motivi). È dunque proprio la presenza del punto a segnalare l'autonomia illocutiva delle due unità.

Dall'altro lato, invece, si hanno esempi in cui l'unità introdotta da perché risulta sintatticamente e pragmaticamente indipendente rispetto al cotesto precedente a prescindere dalla configurazione interpuntiva che la caratterizza. Questo accade ad esempio quando reggente e causale corrispondono ad atti illocutivi di tipo diverso, come in (8), in cui si hanno un atto espressivo e uno assertivo:

(8) Come non passa il tempo. Perché è Luca Cantagalli quello che rivolta la finale, nel quarto set, per l'ultima volta, dando il ventesimo scudetto a 
Modena, in tre partite e soffocando le estreme illusioni dei campioni in carica di Treviso. (PUNT-IT_261_Repubblica_30.04.1995_Sport)

Perché esprime un atto di motivazione autonomo, che si aggancia all'atteggiamento illocutivo, come in (8) o, in altri casi, epistemico; e proprio quest'ultima realizzazione è la più comune.

$\mathrm{Si}$ tratta di manifestazioni che ricordano gli usi di causali autonome menzionati per il parlato (cfr. $§ 2$ ). È noto infatti che la scrittura giornalistica odierna assorbe con facilità fenomeni e strategie linguistiche tipiche del parlato comune. Se però nell'oralità interviene la prosodia a distinguere questa configurazione grazie a confini intonativi terminali, in questo caso il ruolo chiave è giocato dal segno di punteggiatura, che proietta in modo evidente il confine forte, enunciativo. Una differenza significativa tra le due varietà per quanto concerne il fenomeno delle causali indipendenti riguarda senz'altro la frequenza di occorrenza: gli studi su perché nel parlato considerano questa manifestazione tra le più comuni (cfr. Acciardi 2010), mentre nello scritto essa non corrisponde a uno degli sfruttamenti prototipici, ma, ciononostante, è risultata nel corpus molto più diffusa di quanto atteso. Va inoltre sottolineato come nella scrittura controllata, sottoposta a operazioni di rilettura e revisione, lo scrivente che sceglie di adottare tale strategia testuale lo faccia in modo più consapevole dello scarto che questa crea rispetto alla norma - che non menziona l'uso in enunciato autonomo tra le possibili manifestazioni della causale (cfr. ad esempio Serianni 1989) -, con l'intenzione di provocare l'effetto interpretativo descritto.

\section{Weil come introduttore di enunciati autonomi: un'analisi nei giornali tedeschi}

Dopo aver delineato la diffusione e le funzioni delle causali che compaiono in un enunciato autonomo in italiano, si apre ora all'ottica contrastiva, adottando come lingua di paragone il tedesco e come varietà di scrittura sempre quella giornalistica. L'obiettivo è verificare se - con che frequenza e quali valori - le combinazioni delle causali con usi interpuntivi più estremi e guidati, come abbiamo mostrato, da ragioni d'essere puramente comunicative siano penetrati anche nella realtà dell'uso di una lingua la cui la punteggiatura in generale è regolata da vincoli fortemente sintattici, come viene spiegato di seguito: 
Es wird «beabsichtigt, nicht nur das Komma sondern alle Interpunktionszeichen als syntaktische Mittel zu erfassen. (...) Die Interpunktion als syntaktisches Mittel angemessen beschrieben ist ${ }^{7} \gg$. (Behrens 1989: 16; in Bredel 2009: 117)

Per comprendere il fondamento sintattico dell' uso interpuntivo tedesco, basti pensare alla regola che prevede l'inserzione della virgola sistematicamente prima di ogni subordinata, ignorando le distinzioni informative che le caratterizzano, persino nel caso dell'opposizione tra relative restrittive e relative appositive. Si tratta di un'impostazione fortemente diversa da quella italiana - ma anche francese, spagnola, inglese -, in cui come mostrato in Ferrari et al. (2018) l'uso dei diversi segni interpuntivi segue un principio comunicativo-testuale, contribuendo, ad esempio, alla segmentazione del testo nelle sue unità comunicative costitutive e alla loro gerarchizzazione. Ciò è stato mostrato collateralmente nel $\S 2.2$ a proposito dell'uso della virgola con perché. Vista l'impostazione sintattica dell'interpunzione tedesca, si potrebbe ipotizzare che usi del punto "estremi" come quelli mostrati per l'italiano $(\S 2.3)$ entrino con maggiore difficoltà nella realtà della scrittura.

L'indagine preliminare che si propone in queste pagine si sofferma in particolare sul connettivo weil nella funzione di introduttore di causali che si collocano in enunciato autonomo. Tra i diversi connettivi subordinanti causali disponibili in tedesco - come da, um so mehr als, um so weniger als, zumal-esso è riconosciuto come Allzweckkonnektor (Frohning 2007: 136), costituendo l'equivalente funzionale dell'italiano perché, adottato come caso paradigmatico nei paragrafi precedenti ( $\$ 2.2$ e $\$ 2.3$ ). Studi sul connettivo tedesco (cfr. per un’analisi dettagliata sulle funzioni di weil Breindl et al. 2014: 840-858 e sul confronto i connettivi italiani Ravetto \& Blühdorn 2011 e Ballestracci 2011), infatti, hanno già mostrato come esso in termini di frequenza sia l'introduttore causale più diffuso nella scrittura giornalistica; a livello sintattico preferisca la posizione posposta; a livello informativo si possa inserire in tutte le configurazioni elencate in precedenza a proposito di perché; e infine a livello semantico presenti la stessa flessibilità di quest'ultimo, potendo introdurre relazioni che si agganciano al contenuto proposizionale, epistemico o illocutivo. Date le proprietà che caratterizzano il connettivo e lo rendono vicino a perché, la subordinata con weil sembrerebbe avere $\mathrm{i}$ requisiti per presentarsi anche in isolamento nel testo. Ed effettivamente fenomeni di emarginazione sono già riportati in letteratura come casi di non integrazione

\footnotetext{
${ }^{7}$ Trad.: 'Tutti i segni di punteggiatura, non solo la virgola, sono da considerare come mezzi sintattici. La punteggiatura è descritta adeguatamente come mezzo sintattico'. Le traduzioni delle citazioni e degli esempi tedeschi che si propongono in questa sezione sono da intendere come traduzioni di servizio.
} 
sintattica; ad esempio, in Breindl \& Walter (2009: 58) si specifica che: «Wenn eine deutlich markierte Grenze wie beispielsweise ein Satzschlusszeichen vorliegt $[\ldots]$, wurde dies als Desintegrationssignal gewertet $[\ldots]^{8} \gg$.

Per effettuare un sondaggio preliminare e verificare l'effettiva portata del fenomeno, si è selezionato un campione di circa 430 causali introdotte da weil, ovvero lo stesso numero di casi individuati e analizzati per perché (\$ 2.1), in modo da avere a disposizione un bacino quantitativamente paragonabile e poter introdurre alcune prime considerazioni quantitative a confronto tra le due lingue. Per quanto riguarda la varietà testuale, si è attinto dalle sezioni di prosa giornalistica dell'ampissimo corpus di tedesco scritto DeReko, che copre svariate Textsorten ${ }^{9}$, per un totale di circa 43 miliardi di parole, costituendo il più grande archivio di tedesco scritto contemporaneo. In particolare, gli esempi provengono da tre delle numerose testate disponibili: Die Zeit, Süddeutsche Zeitung e Tagezeitung, tra i quotidiani tedeschi con un più alto numero di tirature. Le occorrenze, spartite in modo equo tra $\mathrm{i}$ tre giornali, sono state raccolte a partire da articoli selezionati in modo random, relativi a diverse rubriche, e riconducibili all' arco temporale che va dal 2015 al $2018^{10}$.

Lo spoglio di questi testi ha sì confermato la presenza nei giornali tedeschi di causali che esauriscono un enunciato, come la seguente:

(9) [Er] nannte Ottl bald: den Schwamm. Weil der sich umschaute Beobachtete. Nachdachte. (U15/JAN.00031 Süddeutsche Zeitung, 02.01.2015, p. 31)

'Presto chiamò Ottl: la spugna. Perché si guardava intorno. Osservava. Rifletteva.'

Tuttavia, ha fatto emergere un dato meno forte rispetto a quello ottenuto per l'italiano (cfr. § 2.3), confermando così l'ipotesi iniziale: in tedesco il fenomeno appare più marcato. In particolare, sulla totalità delle occorrenze in posizione

\footnotetext{
${ }^{8}$ Trad.: 'Quando si ha un confine marcato in modo chiaro, ad esempio un segno interpuntivo che indica la fine della frase, questo è stato valutato come segnale di non integrazione'.

${ }^{9} \mathrm{Il}$ corpus contiene in particolare: «belletristische, wissenschaftliche und populärwissenschaftliche Texte, eine große Zahl von Zeitungstexten sowie eine breite Palette weiterer Textarten und werden kontinuierlich weiterentwickelt» (http://www1.ids-mannheim.de/kl/projekte/korpora.html). Per un approfondimento, cfr. Kupietz \& Keibel (2009).

${ }^{10}$ Più precisamente, sono stati scelti quattro numeri (del mese di gennaio) per ogni quotidiano, uno per ogni annualità.
} 
posposta ( $91 \%$ delle causali raccolte $\left.{ }^{11}\right)$ la causale compare in enunciato autonomo in circa il 13\% dei casi. Nell'ambito della scrittura giornalistica, la percentuale più bassa del tedesco andrebbe a confermare le impressioni già avanzate in studi precedenti (cfr. ad esempio Costa 2011), secondo cui la portata del fenomeno delle emarginazioni di frasi o sintagmi a opera della punteggiatura forte complessivamente - non soltanto in riferimento al costrutto causale - è più alta nei giornali italiani.

Tra i fattori che possono incidere sulla percentuale più bassa si ha il fatto che in tedesco, restando nell'ambito della frase complessa, esiste un costrutto alternativo che può competere con quello inaugurato da weil nella manifestazione enunciativamente autonoma, ovvero la frase introdotta da denn:

(10) Die Entlassungen sind nicht die Konsequenz, sie sind das Symptom der Krise. Denn ein Trainerwechsel ist nur dann sinnvoll, wenn der Trainer in ein funktionierendes Umfeld kommt. Auch das zeigt sich in Hamburg. (Z15/JAN.00036 Die ZEIT, 08.01.2015, p. 2)

'I licenziamenti non sono la conseguenza, sono il sintomo della crisi. Perché un cambio di allenatore è utile solo se l'allenatore arriva in un ambiente che funziona.'

In generale, per via delle funzioni semantico-pragmatiche associate al connettivo denn - seguendo Breindl et al. (2014: 866-874) e Ravetto \& Blühdorn (2011) la sovrapposizione con weil potrebbe avvenire per i casi in cui la subordinata è posposta, focalizzata in modo autonomo rispetto alla sua conseguenza e introduce una relazione legata all'atteggiamento epistemico o illocutivo. Si tratta di condizioni che, come mostreremo nel $\S 3.1$, si realizzano spesso per gli usi della causale enunciata in modo autonomo. A livello morfosintattico, visto che lo statuto di denn è più vicino a quello delle Konjunktionen (che delle Subjunktionen), la forma verbale principale dell'enunciato occupa la seconda posizione sintattica come nelle frasi principali. La frase causale con denn è dunque una frase indipendente a tutti gli effetti, che si comporta in modo simile al secondo membro di una coordinazione. Per quanto riguarda la manifestazione in enunciato indipendente, si è potuto osservare grazie a una ricognizione corpus-based l'effettiva facilità di denn a essere preceduto dal punto fermo: a partire dallo stesso numero di dati e dalle stesse testate giornalistiche di DeReKo osservate per weil, la percentuale di casi in cui la frase causale con denn è preceduta dal punto, anziché dalla virgola, arriva

\footnotetext{
${ }^{11} \mathrm{Si}$ nota collateralmente che la subordinata con weil occorre con maggiore facilità in posizione pre-reggente rispetto a quella con perché, che appare, come osservano anche Ravetto/Blühdorn 2011: 244, più marcata se anteposta.
} 
addirittura al $60 \%$, risultando persino la configurazione interpuntiva più usata in combinazione con questo Konnektor. Dunque, qualora lo scrivente desideri esprimere una motivazione in enunciato autonomo tende a selezionare questa struttura. Affinché si possa avere una visione completa del fenomeno della frase causale in enunciato autonomo in tedesco occorrerebbe approfondire il confronto tra questi due connettivi causali.

\subsection{Usi del punto tra reggente e causale}

Mantenendo il focus solo sulle causali con weil, si osserva che il dato presentato nel paragrafo precedente (il 13\% del totale delle occorrenze indagate) necessita di essere ulteriormente disaggregato. L'analisi dei singoli esempi ha infatti permesso di distinguere due possibilità, che si spartiscono in modo equo il bacino totale di causali enunciativamente autonome. Da un lato, si trovano occorrenze in cui il punto prima del connettivo si colloca effettivamente tra reggente e causale, come (11), in cui si ha una struttura del tipo 'p. Weil q':

(11) Mittlerweile ist sie Professorin für Literaturwissenschaft an der Universität Stockholm, spezialisiert auf Verfasser wie Gustave Flaubert, Marcel Proust, James Joyce und Thomas Mann. Weil "ihr Werk zeigt, wie stilistische Veränderungen mit politischen und gesellschaftlichen zusammenhängen". (T15/JAN.00011 die tageszeitung, 02.01.2015, p. 02) 'Adesso è professoressa di letteratura all'Università di Stoccolma, specializzata in autori come Gustave Flaubert, Marcel Proust, James Joyce e Thomas Mann. Perché "il loro lavoro mostra come i cambiamenti stilistici sono collegati a quelli politici e sociali".'

Queste si alternano a occorrenze in cui il segno forte si colloca tra due causali giustapposte o coordinate tra loro, come accade in (12) col punto prima di Und weil:

(12) Aber es ist eben der 2. Januar, der sich als Schicksalstag ins kollektive Gedächtnis der Stadt eingebrannt hat. Weil an diesem Tag die bedeutendste Altstadt des mittelalterlichen Heiligen Reichs zertrümmert wurde. Und weil es ein Angriff über Nacht war. (U15/JAN.00032 Süddeutsche Zeitung, 02.01.2015, p. 33)

'Perché quel giorno il centro storico più importante del Sacro Impero medievale fu distrutto. E perché si è trattato di un attacco avvenuto durante la notte.' 
Come si nota, i due casi precedenti costituiscono due differenti manifestazioni, che potrebbero essere ricondotte a diverse ragioni d'essere, che saranno tratteggiate di seguito.

A livello interpuntivo, il segno forte che precede weil in entrambe le configurazioni è sistematicamente il punto; se infatti nel corpus italiano costituiscono un'alternativa abbastanza diffusa i due punti, in tedesco non si sono individuati sfruttamenti simili del segno ${ }^{12}$.

Da questo primo quadro ne deriva, dunque, non solo che il dato relativo ai casi di weil che introduce causali in enunciato autonomo è più basso di quello emerso in italiano per perché, ma anche che in quasi la metà delle occorrenze non siamo di fronte agli usi effettivamente comunicativi del punto che interviene a separare reggente e subordinata ma a usi in cui il segno assume piuttosto una funzione enumerativa, andando ad articolare una sequenza di subordinate.

Per quanto riguarda gli usi in cui il punto si inserisce tra reggente e causale, se a livello quantitativo è emerso un dato più basso rispetto a quello ricavato in italiano, a livello funzionale invece, il comportamento delle emarginazioni frasali tedesche appare molto vicino a quello delle causali italiane con perché. Il fenomeno sembra avere ragioni di essere comunicativo-testuali: focalizzare in modo evidente la causa/motivazione. Più in particolare, in linea con quanto mostrato per l'italiano, si riconoscono due possibilità a livello interpretativo. Si hanno esempi in cui il punto va a inserirsi in un costrutto altrimenti potenzialmente coeso a livello sintattico-semantico, rendendo causale e reggente indipendenti testualmente, come in (13):

(13) [sie] ist [...] spezialisiert auf Verfasser wie Gustave Flaubert, Marcel Proust, James Joyce und Thomas Mann. Weil "ihr Werk zeigt, wie stilistische Veränderungen mit politischen und gesellschaftlichen zusammenhängen". (T15/JAN.00011 die tageszeitung, 02.01.2015, p. 02) 'È specializzata in autori come Flaubert, Joyce, Proust e Mann. Perché "il loro lavoro mostra come i cambiamenti stilistici sono collegati a quelli politici e sociali".'

\footnotetext{
${ }^{12}$ Per completezza, occorrerebbe condurre anche un'analisi sui casi di subordinate causali che seguono un Gedankenstrich, che qui non abbiamo considerato. In tedesco questo segno è usato più spesso che in italiano e potenzialmente anche in contesti che si sovrappongono con quelli per cui in italiano useremmo proprio i due punti; cfr. ad esempio Longo \& Stojmenova Weber (2019). Per un'analisi sistematica degli usi della lineetta in tedesco si rimanda ai lavori di Marcella Costa, ad esempio Costa $(2011,2017)$.
} 
Se al posto del punto avessimo una virgola si sarebbe potuto interpretare il costrutto come più integrato sintatticamente. Sempre nell'ambito di questa prima possibilità, accanto a esempi come (13), in cui la frase con weil emarginata tra due punti forti si alterna a enunciati tradizionali, saturati da frasi semplici o complesse, se ne hanno altri in cui lo style coupé (cfr. Bonomi 2002) risulta una marca stilistica dello scrivente, che lo adotta in modo sistematico nello stesso articolo, come in (14):

(14) [Er] nannte Ottl bald: den Schwamm. Weil der sich umschaute. Beobachtete. Nachdachte. [...]. Und weil er sich so in den fünfeinhalb Spielzeiten für den FC Bayern den Ruf eines Musterprofis erwarb. Er trainierte fleißig. Er maulte nie. Und wenn ein Spieler verletzt war oder geschont wurde, spielte Ottl. Aufmerksam, solide, unaufgeregt. (U15/JAN.00031 Süddeutsche Zeitung, 02.01.2015, p. 31)

'Presto chiamò Ottl: la spugna. Perché si guardava intorno. Osservava. Rifletteva. E perché ha guadagnato nelle cinque stagioni e mezzo con il Bayern la reputazione di professionista modello. Si allenava duramente. Non si lamentava mai. E se un giocatore era infortunato o veniva risparmiato, Ottl giocava. In modo attento, solido, tranquillo.'

Il brano riportato costituisce un caso esemplare del periodare monoproposizionale descritto per lo stile giornalistico italiano (cfr. $§ 2.3$ ). In particolare, si ha sia sintassi franta (cfr. Bonomi 1993) - sequenze di enunciati saturati da subordinate causali, da frasi dipendenti o indipendenti coordinate o ancora da semplici sintagmi verbali o avverbiali isolati tra punti - sia successioni di frasi semplici e molto brevi.

Questi casi (13)-(14), sulla totalità delle manifestazioni del punto tra reggente e causale con weil, sono i meno comuni nel corpus, in linea con quanto osservato anche per l'italiano. Nella maggior parte degli esempi siamo di fronte a costrutti in cui le due proposizioni risultano illocutivamente autonome a prescindere dal segno interpuntivo che interviene a separarle, come in (15) e (16):

(15) Schließlich haben sie überlebt! Weil jeder Brüche in seinem Leben aufweist. (U16/JAN.00058 Süddeutsche Zeitung, 02.01.2016, p. 14) 'Dopo tutto sono sopravvissuti! Perché ognuno ha delle fratture nella vita.'

(16) Das ist ein Vergraben in alle Richtungen. Weil sich die Menschen nicht mehr respektieren und nicht mehr füreinander interessieren. Ganz viele sind nur noch damit beschäftigt, irgendwie über die Runden zu kommen und ihr Ding zu machen, auf sich zu schauen. Die Prioritäten haben sich 
bei vielen sehr verschoben. Vielleicht hat es auch mit einer gewissen Dankbarkeit und Demut zu tun, die ich überhaupt nicht mehr spüre bei den Menschen. (U18/JAN.00515 Süddeutsche Zeitung, 05.01.2018, p. 36) 'È un sotterramento in tutti i sensi. Perché le persone non si rispettano più e non sono più interessate l'una all'altra. Molte persone cercano solo di sbarcare il lunario in qualche modo e fare le loro cose, pensare a se stesse. Le priorità per tanti sono molto cambiate.'

A livello semantico, siamo qui di fronte a relazioni di composizione testuale: in particolare con la causale in (15) ci si aggancia al contenuto illocutivo della reggente per motivare il fatto di aver compiuto il particolare atto espressivo; e con quella in (16) si introduce una motivazione argomentativo-esplicativa con cui si giustifica l'opinione espressa in precedenza. Sempre in linea con l'italiano, quest'ultimo caso risulta il più comune: la causale in enunciato autonomo è utilizzata soprattutto per motivare opinioni, ipotesi, ovvero come strategia argomentativa. A questo proposito, si è potuto confermare che anche in tedesco la causale autonoma si trova spesso ad aprire movimenti argomentativi con portata ampia verso il cotesto destro, che vanno a coinvolgere più enunciati, se non l'intero capoverso. È ciò che accade nell'esempio (16), in cui il tema introdotto nella causale (weil sich die Menschen nicht mehr respektieren und nicht mehr füreinander interessieren) viene motivato e specificato negli enunciati successivi. Altre particolari forme di motivazione estesa saranno illustrate nel $\S 3.2$.

Finora si sono messi in evidenza diversi punti di contatto a livello semanticotestuale tra l'uso in tedesco e in italiano di questi costrutti marcati. Un fattore di discrepanza riguarda, invece, il livello morfosintattico, e in particolare la posizione del verbo principale nella frase. Diversamente da quanto accade in italiano, nel tedesco scritto standard la causale, come ogni altra subordinata, si distingue dalle frasi indipendenti anche per la collocazione del verbo: in posizione conclusiva della frase anziché nella seconda posizione sintattica (Er kommt nicht. Weil es zu spät ist). Tuttavia, è ormai notata anche dalla norma grammaticale (cfr. Duden 2009) la tendenza di alcuni tipi di subordinate - compresa la causale con weil - a mantenere la distribuzione del verbo prevista per le frasi principali ( $\mathrm{Er}$ kommt nicht. Weil es ist zu spät) in specifici contesti d'uso. Seguendo Breindl et al. (2014: 840-843), il fenomeno si manifesta, in contesti substandard, ovvero nel parlato e in alcuni dialetti, ma è individuato qua e là anche nello scritto, soprattutto per mettere in atto mimesi del parlato; a livello sintattico e pragmatico, solo quando la causale è posposta, non integrata, ma focalizzata in modo indipendente rispetto alla reggente (anch'essa focalizzata), e agganciata a livello epistemico o illocutivo. Per quanto riguarda lo specifico caso della causale con weil collocata 
in enunciato autonomo, anche qualora si realizzino le condizioni appena elencate, la posizione del verbo negli esempi osservati rimane quella standard. Dunque, nonostante l'autonomia sintattico-testuale acquisita dalle causali considerate, che occorrono in un enunciato indipendente, e nonostante il fatto che si tratti di strutture non canoniche per lo scritto e anzi più vicine a certi usi delle subordinate autonome del parlato, esse mantengono la caratteristica morfologica distintiva delle frasi dipendenti. Questo potrebbe essere un segno della maggiore difficoltà del tedesco a plasmare la struttura morfosintattica ai dettati della comunicazione.

\subsection{Sequenze di causali autonome}

Come notato nel paragrafo precedente, la subordinata causale autonoma tende ad aprire movimenti argomentativi con portata ampia verso gli enunciati successivi. A livello di espressione linguistica, ciò si traduce spesso in sequenze di causali giustapposte o coordinate, come quelle esemplificate di seguito:

(17) Aber es ist eben der 2. Januar, der sich als Schicksalstag ins kollektive Gedächtnis der Stadt eingebrannt hat. Weil an diesem Tag die bedeutendste Altstadt des mittelalterlichen Heiligen Reichs zertrümmert wurde. Und weil es ein Angriff über Nacht war. (U15/JAN.00032 Süddeutsche Zeitung, 02.01.2015, p. 33)

'Ma è il 2 gennaio che è rimasto impresso nella memoria collettiva della città come giorno fatidico. Perché quel giorno il centro storico più importante del Sacro Impero Medievale fu distrutto. E perché si è trattato di un attacco avvenuto durante la notte.'

(18) Dieses Glück, das nur der glitzernde Schnee und die Berge bei Sonnenschein liefern, es bleibt unbelastet von Gewissensbissen (wegen der Umwelt und des vielen Kaiserschmarrns) und Kostenkalkulationen (wegen der Hotelkosten und Skipässe). Weil der Kick einer scharf gefahrenen Kurve, die man gerade noch so gepackt hat, in Euphorie umschlägt, wenn der Berg flacher ausläuft. Weil regelmäßig Dopamin durch den Körper flutet, dazu die tolle Aussicht. Weil die Schenkel am zweiten Tag so beißen, dass man förmlich zu spüren glaubt, wie der Kaiserschmarrn verbrannt wird. Und weil man halt so gut schläft, nach einem Skitag. (U16/JAN.00053 Süddeutsche Zeitung, 02.01.2016, p. 11) 'Perché il brivido di una salita fatta duramente si trasforma in euforia quando la montagna appare più piatta. Perché la dopamina fuoriesce regolarmente dal corpo, con la vista fantastica. Perché il secondo giorno le cosce fanno così male che sembra quasi di avere la sensazione che il 
Kaiserschmarrn venga bruciato. E perché si dorme così bene dopo una giornata di sci.'

Tipicamente si hanno più causali in successione - fino addirittura a quattro, come in (18) -, ciascuna delle quali satura un enunciato separato dal precedente per mezzo del punto fermo.

In qualche caso invece soltanto l'ultima causale della serie è collocata in un enunciato distinto da quello in cui compaiono le altre causali (o l'altra causale) a cui è coordinata, come in (19):

(19) [sie] hat sich im November für den Chor angemeldet, weil sie unbedingt zum Dom wollte an Silvester. [...]. Und weil dort diesmal nicht geknallt werden durfte. Das vergangene Jahr hat etwas verändert in der Gesellschaft und in ihr, sagt sie: Es gibt mehr Misstrauen dem Fremden gegenüber. (U17/JAN.00036 Süddeutsche Zeitung, 02.01.2017, p. 3) 'Si è iscritta al coro a novembre perché voleva assolutamente andare in cattedrale a San Silvestro. [...]. E perché questa volta non ci sarebbero potuti essere scoppi. Lo scorso anno ha cambiato qualcosa nella società e in lei, dice: c'è più sfiducia nei confronti dello straniero.'

In ottica contrastiva, l'analisi ha permesso di ricavare un dato indicativo. Sebbene sequenze di causali giustapposte siano state osservate anche nel corpus italiano, come mostra (20), in cui a scandire i singoli membri della serie è, questa volta, il punto e virgola:

(20) La seconda Tangentopoli - ormai è d'uso chiamare così l'affare Necci - è davvero più grave della prima. Perché spazza gli alibi con cui erano state addossate tutte le responsabilità ai politici [...]; perché solleva il velo su ampi settori dell'economia che non erano stati toccati [...]; perché autorizza seri dubbi sui rimedi e sulle vie d'uscita che erano stati individuati. (PUNT-IT_222_Corriere_della_Sera_25.09.1996_Editoriali; in Rosi 2018)

Proporzionalmente, questi usi appaiono più diffusi nei giornali tedeschi. In questa lingua infatti essi costituiscono uno degli sfruttamenti più comuni della causale in enunciato autonomo e incidono così, come si è mostrato, in modo decisivo sul computo complessivo ricavato (quasi la metà delle causali autonome è inserita infatti in sequenze giustapposte; cfr. $\$ 3.1$ ).

A livello interpretativo, si può ipotizzare che il fatto di distribuire le causali in enunciati autonomi e distinti anziché collocarle in un unico complesso enunciato 
possa essere ricondotto al desiderio dello scrivente di semplificare la struttura sintattica del periodo. Si evita cioè di creare enunciati troppo lunghi e articolati, con all'interno più subordinate coordinate; il che va a semplificare anche il processo di lettura e di interpretazione dell'articolo. Se così fosse, si confermerebbe la tendenza del tedesco a fare un uso sintattico della punteggiatura persino nei suoi impieghi più marcati. Ciò detto, non si esclude però che collateralmente alcune manifestazioni del fenomeno possano avere anche ricadute comunicative: ad esempio, in (19) l'isolamento solo dell'ultima causale della serie può provocare un effetto di maggiore focalizzazione rispetto alle precedenti, che ne faciliterebbe anche la tematizzazione nel cotesto susseguente.

\section{Conclusioni}

L'indagine proposta ha permesso, in primis, di delineare un quadro dell'uso contemporaneo delle causali nell' italiano giornalistico, e inoltre di confrontarne alcune manifestazioni meno canoniche con quelle corrispondenti nella stampa tedesca. Per quanto riguarda l'italiano, adottare un approccio corpus-based, che ha fatto interagire indagini quantitative e qualitative, ha permesso di riconoscere alcuni punti di vicinanza tra l'uso delle strutture causali nella varietà testuale considerata specchio della scrittura standard odierna e l'uso descritto in letteratura per il parlato-parlato, in particolare per quanto riguarda la scelta dei diversi nessi introduttori di causalità. Le due varietà diamesiche sono infatti accomunate da uno scarso sfruttamento delle potenzialità offerte a livello di sistema, a favore quasi di un unico mezzo: il connettivo perché. L'analisi di perché ha intrecciato i livelli sintattico, semantico-concettuale e informativo-testuale, mostrandone la reciproca interdipendenza. È emerso, ad esempio, che le causali aperte dal connettivo offrono tendenzialmente un importante contributo all'architettura semanticopragmatica del testo nei giornali indagati, risultando rilevanti in termini comunicativi. Tale rilievo diviene massimo quando esse si collocano in enunciati distinti, separate da un segno interpuntivo forte; configurazione che è risultata ormai diffusa nella stampa italiana. Proprio quest'ultima manifestazione testuale meno prototipica è stata oggetto del confronto in ottica contrastiva. Anche in tedesco il fenomeno della causale autonoma è presente. In termini quantitativi, però, almeno nel bacino di esempi considerato, appare più marcato. Più marcato è anche a livello formale e funzionale: il punto prima di weil si colloca tra reggente e causale solo in un sottoinsieme delle occorrenze; in altri casi assume una funzione sintattico-enumerativa, andando ad articolare sequenze di causali giustapposte. A livello morfosintattico, infine, la posizione del verbo nella causale resta quella 
finale, tipica delle subordinate tedesche, nonostante l'autonomia enunciativo-testuale acquisita da questi costrutti.

\section{Bibliografia}

Acciardi, D. 2010. La subordinazione causale esplicita nel parlato. Un'analisi corpus-based (CORAL-ROM Italia). In V. Bambini, I. Ricci \& P.M. Bertinetto (eds), Linguaggio e cervello/Semantica. Atti del XLII Congresso internazionale di Studi SLI (Pisa, Scuola Normale Superiore, 25-27 settembre 2008), vol. 2 (CD ROM). Roma: Bulzoni.

Antonelli, G. 2011. Lingua. In A. Afribo \& Emanuele Zinato (eds), Modernità italiana. Cultura, lingua e letteratura dagli anni settanta a oggi. Roma: Carocci, 15-52.

Ballestracci, S. 2011. Die kausalen Verknüpfungsmittel des Deutschen und des Italienischen. Eine konstrastive Beschreibung unter formalem und funktionalem Aspekt. Linguistik online 49(5/201): 75-89.

Behrens, U. 1989. Wenn nicht alle Zeichen trügen. Interpunktion als Markierung syntaktischer Konstruktionen. Frankfurt: Lang.

Berretta, M. 1994. Il parlato italiano contemporaneo. in L. Serianni \& P. Trifone (eds), Storia della lingua italiana 2: Scritto e parlato. Torino: Einaudi, 239-270.

Bonomi, I. 1993. I giornali e l'italiano dell'uso medio. Studi di Grammatica italiana XV: 81201.

Bonomi, I. 2002. L'italiano giornalistico. Dall'inizio del '900 ai quotidiani on line. Firenze: Franco Cesati.

Breindl, E., Volodina, A. \& Waßner, U. H. 2014. Handbuch der deutschen Konnektoren 2. Semantik der deutschen Satzverknüpfer. Berlin-New York: de Gruyter.

Breindl, E. \& Walter, M. 2009. Der Ausdruck von Kausalität im Deutschen. Eine korpusbasierte Studie zum Zusammenspiel von Konnektoren, Kontextmerkmalen und Diskursrelationen. Manheim: Instituit für Deutsche Sprache (= amades. Arbeiten und Materialen zur deutschen Sprache 38).

Bredel, U. 2009. Das Interpunktionssystem des Deutschen. In A. Linke \& H. Feilke (eds), Oberfläche und Performanz. Untersuchungen zur Sprache als dynamischer Gestalt. Tübingen: Niemeyer, 117-136.

Costa, M. 2011. Interpunktionspraktiken im Vergleich - am Beispiel von Punkt und Gedankenstrich. In S. B. Coletsos, M. Costa \& L. Eichinger (eds), Deutsch-Italienisch. Sprachvergleiche. Heidelberg: Winter, 167-188.

Costa, M. 2017. L'interpunzione come segnaletica testuale nel tedesco contemporaneo In A. Ferrari, L. Lala \& F. Pecorari (eds), L'interpunzione oggi (e ieri). L'italiano e altre lingue europee. Firenze: Franco Cesati, 307-324.

Cresti, E. 2012. L'unité de suffixe : identification et interprétation des unités de la langue parlé In S. Caddéo, M. N. Roubaud \& M. Rouquier \& F. Sabio (eds), Penser les langues avec Claire Blanche-Benveniste. PUP: 201-213.

Duden. 2009. Die Grammatik. Mannheim/Leipzig/Wien/Zürich: Dudenverlag.

Duden. 2018. Komma, Punkt und alle anderen Satzzeichen, Das Handbuch Zeichensetzung. Mannheim/Leipzig/Wien/Zürich: Dudenverlag.

Ferrari, A. 1995. Connessioni. Uno studio integrato sulla subordinazione avverbiale. Ginevra: Slatkine. 
Ferrari, A. 2016. La subordinazione sintattica e la subordinazione informativa, tra isomorfismo e non isomorfismo. In P. D'Achille (ed.), Grammatica e testualità. Metodologia ed esperienze didattiche a confronto. Firenze: Cesati, 41-54.

Ferrari, A., Cignetti, L., Cesare, A. M., Lala, L., Mandelli, M., Ricci, C. \& Roggia, C. E. 2008. L'interfaccia lingua-testo. Natura e funzioni dell'articolazione informativa dell'enunciato. Alessandria: Edizioni dell'Orso.

Ferrari, A., Lala, L., Longo, F., Pecorari, F., Rosi, B. \& Stojmenova, R. 2018. La punteggiatura italiana contemporanea. Un'analisi comunicativo-testuale. Roma: Carocci.

Ferrari, A. \& Rosi, B. in stampa. Virgola e subordinate circostanziali nella scrittura contemporanea. Lingua e stile.

Frohning, D. 2007. Kausalmarker Zwischen Pragmatik Und Kognition. Boston-Berlin: De Gruyter.

Kupietz, M. \& Keibel, H. 2009. The Mannheim German Reference Corpus (DeReKo) as a basis for empirical linguistic research. In M. Minegishi (ed.), Working Papers in Corpus-based Linguistics and Language Education 3. Tokyo: Tokyo University of Foreign Studies (TUFS), 53-59.

Longo, F. \& Stojmenova Weber, R. 2019. L'intercambiabilità della lineetta singola e dei due punti in testi tradotti e non tradotti. In A. Ferrari, L. Lala, F. Pecorari \& R. Stojmenova Weber (eds) Punteggiatura, sintassi, testualità nella varietà dei testi italiani contemporanei. Firenze: Franco Cesati, 455-468.

Lüger, H. H. 1983. Pressesprache. Tübingen: Max Niemieyer Verlag (Germanistische Arbeitshefte 28).

Moeschler, J. 1987. Trois emplois de parce que en conversation. Cahiers de Linguistique Française 8: 97-110.

Panunzi, A. \& Gregori, L. 2012. DB-IPIC. An XML Database for the Representation of Information Structure in Spoken Language. In H. Mello, A. Panunzi \& T. Raso (eds), Pragmatics and Prosody. Illocution, Modality, Attitude, Information Patterning and Speech Annotation. Firenze: Firenze University Press, 133-150.

Previtera, L. 1996. I costrutti causali. Studi italiani di linguistica teorica e applicata 25(1): 2946.

Ravetto, M. \& Blühdorn, H. 2011. Die Kausalkonjunktionen denn, weil, da im Deutschen und perché, poiché, siccome im Italienischen. In G. Ferrarese (ed.), Konnektoren im Deutschen und im Sprachvergleich. Beschreibung und Gramatische Analys., Tübingen: Narr, 207-250.

Rosi, B. 2018. Le (complesse) interazioni tra sintassi e punteggiatura: le subordinate causali introdotte da perché. In A. Ferrari, L. Lala, F. Longo, F. Pecorari, B. Rosi \& R. Stojmenova Weber (eds), La punteggiatura italiana contemporanea. Un'analisi comunicativo-testuale. Roma: Carocci, 35-48.

Rosi, B. 2019. La causalità tra subordinazione e giustapposizione nell'italiano contemporaneo scritto e parlato. Ph.D. diss., Universität Basel.

Rosi, B. \& Ferrari, A. 2019. Il costrutto causale nello scritto, tra grammatica, linguistica e didattica. In V. Carbonara, L. Cosenza, P. Masillo, L. Salvati \& A. Scibetta (eds), Il parlato e lo scritto: aspetti teorici e didattici, Pisa: Pacini Editore, 17-27.

Serianni, L. 1989. Grammatica italiana. Italiano comune e lingua letteraria. Torino: UTET.

Sweetser, E. 1990. From etimology to pragmatics. Cambridge: Cambridge University Press.

Voghera, M. 2017. Dal parlato alla grammatica. Roma: Carocci. 